\title{
INSIDE LAB INVEST
}

\section{MAKING INROADS IN THE MOLECULAR BASIS OF HEREDITARY MACRO-THROMBOCY-}

TOPENIAS: Mutations in the MYH9 gene, encoding the nonmuscle myosin heavy chain A (NMMHCA) cause a series of diverse clinical syndromes characterized by giant platelets, thrombocytopenia, and cytoplasmic inclusion bodies in granulocytes. Using two antibodies directed against different regions of the NMMHCA protein, Kunishima et al studied the granulocytes of 24 patients affected by these rare disorders. Their study, reported in this issue (Lab Invest 2003, 83: 115-122), demonstrates that every patient harboring a mutated allele of the MYH9 gene has an abnormal subcellular localization of NMMHCA. The staining obtained by immunofluorescence coincides with the morphology of the inclusion bodies as revealed by the May-Grunwald-Giemsa stain, and the authors also demonstrate that both the mutated protein and the normal form are present in the inclusion body. This suggests that the product of the mutated MYH9 gene acts as a negative dominant or hypermorph and could explain the autosomal dominant pattern of inheritance seen in these conditions. The examination of granulocytes using antibodies against NMMHCA not only facilitate the diagnosis of patients suffering from these rare disorders, but observation of the subtle morphological differences of the inclusion body allows Kunishima et al to propose three types of staining patterns according to the size, number, and shape of the cytoplasmic inclusions. The notion that the types of inclusion, and perhaps the particulars of the clinical, may be correlated to mutations in particular domains of the protein is not far!

HYPOXIC PRECONDITIONING AND THERAPEUTIC NEOVASCULARIZATION-WHEN LESS RESULTS IN MORE: Recent advances in the isolation, culture, and characterization of embryonic and adult stem cell populations has led to intense interest in using populations of stem cells in the generation of a wide variety of tissues and organs for use as tissue/organ replacements and as progenitor cells capable of repairing particular injured tissues/organs. In light of the magnitude of ischemic vascular disease (both peripheral and cardiac) in the United States and Europe, the application of therapeutic neovascularization is a sought-after approach to a devastating disease affecting great numbers of people. In this issue, Akita et al demonstrate the isolation of human endothelial progenitor cells (EPC) from peripheral blood and their differentiation from peripheral blood mononuclear cells (PB-MNC) in culture (Lab Invest 2003, 83: 65-73). By taking advantage of the fact that hypoxia is known to induce vascular endothelial growth factor (VEGF) production, and VEGF is a potent endothelial differentiation factor and elicites endothelial cell proliferation, the authors were able to stimulate the differentiation of endothelial progenitor cells (EPC) from cultures of peripheral blood mononuclear cells (PB-MNC). Furthermore, they found that this stimulation was sensitive to anti-VEGF antibodies and a VEGF receptor specific tyrosine kinase inhibitor. Finding that hypoxic preconditioning stimulated the differentiation of endothelial progenitor cells as well as their migratory ability prompted the authors to utilize this EPC-enriched preconditioned cell population in a nude rat model of therapeutic neovascularization following the induction of hindlimb ischemia. Interestingly, they noted enhanced neovascularization in the animals receiving the hypoxia-preconditioned cells. This study suggests that the use of a technically simple procedure, hypoxic preconditioning, has the beneficial effects of both stimulating the differentiation of the desired cell type, EPC, and enhancing neovascularization once the cells have been injected into the ischemic area. Additional studies in this area seem warranted to ascertain whether this approach will eventually have widespread application in the area of therapeutic neovascularization and seeding of synthetic and bioengineered vessel segments and heart valves. 